OITS-568

\title{
SELF-ORGANIZED CRITICALITY IN QUARK-HADRON PHASE TRANSITION
}

\author{
Rudolph C. Hwa ${ }^{a}$ and Jicai $\operatorname{Pan}^{b}$ \\ ${ }^{a}$ Institute of Theoretical Science and Department of Physics \\ University of Oregon, Eugene, Oregon 97403 \\ ${ }^{b}$ Department of Physics, McGill University \\ Montreal, Quebec, Canada H3A 2T8
}

\begin{abstract}
The problem of clusters growth in quark-hadron phase transition in heavy-ion collision is investigated by cellular automata. The system is found to exhibit self-organized criticality with the distribution of cluster sizes having universal scaling behavior.
\end{abstract}

This paper describes an unconventional study of an unconventional signature of quark-gluon plasma. It concerns the properties of the hadrons produced after phase transition. It is possible that the proposed signature might be suppressed by factors not yet considered, but if it survives, there are no competing processes that can cause ambiguity in the interpretation of what is observed. For the moment the issue is what to expect under the best of circumstances.

The problem is about the growth of hadronic clusters in the mixed phase, which we take to be in an annular ring in a 2D section at midrapidity of the expanding cylinder after a head-on heavy-ion collision. Hadrons, after nucleation, move radially outward in the annular ring and can encounter one another, resulting possibly in coalescence and in the formation of hadronic clusters, if it is energetically favorable to reduce the surface area. Growth without collisions is also possible. The clusters need not be spherical. Depending on factors that we know very little about dendritic or porous structures cannot be ruled out. Since the clusters in the quark environment behave much like massive colloids suspended in a fluid, they carry out Brownian motion, in the course of which the clusters collide and grow in size. Because 
nucleation can take place anywhere in the mixed region at any time during the period when the mixed phase exists, the clusters emitted at the boundary of the mixed region can have various sizes $S$, depending on how far the nucleation position is from the boundary. A determination of the distribution $P(S)$ of the cluster size would therefore reveal the various aspects of the dynamical process of hadronization mentioned above.

There is no reliable way to treat the cluster growth problem analytically. Our approach is to use cellular automata based on simple rules to simulate the dynamical evolution of the problem. Instead of making drastic approximations necessary to put the problem on a continuum basis amenable to analytical solution, we confront the complications by finding a set of rules that capture the essence of the dynamics. This has been done both in 1D [1] and in 2D [2]. We refer the reader to those references for the details of the precise rules.

In $2 \mathrm{D}$ a wedge of the annular ring containing the mixed region is mapped to a square lattice of $L$ sites in each dimension initially with periodic boundary condition on the upper and lower sides. The nucleation size $S_{0}$ is specified in units of site separation. The complication of confinement at the local level is summarized by one parameter $p$, the nucleation probability. At each time step an unoccupied site (quark) has probability $p$ of becoming occupied (hadron). The occupied sites all take random walks superimposed on an average drift to the right boundary. Clusters of occupied sites become bonded when they meet and become larger clusters. This process is repeated again and again. A cluster of size $S$ reaching the right boundary is taken out of the lattice, and $S$ vertical units of the boundary are moved to the left by one unit. When the moving right boundary reaches the fixed left boundary, the phase transition is over.

Repeated simulation using the automaton thus constructed results in the distribution $P(S)$ that has a power-law behavior

$$
P(S) \propto S^{-\gamma}
$$

as shown in Fig. 1(a), for $L=16, S_{0}=1$, and various values of $p$.

The same behavior persists when $L$ is changed to 32 [Fig. 1(b)], and when $S_{0}$ is changed to 2 [Fig. 1(c)]. Thus the exponent $\gamma$ is independent of $L$ and $S_{0}$, and is rather insensitive to $p$, since for $p$ ranging over an order of magnitude from 0.05 to $0.5, \gamma$ varies only within $10 \%$ of the average value $\gamma \simeq 1.9$. This result on the cluster-size distribution is therefore both scaling and universal. 
Thermal equilibrium has not been assumed. The process is non-equilibrium and dissipative. The system evolves on its own to a critical point characterized by large fluctuations of all sizes. Most significantly, no parameters have been tuned to put the system at the critical point. Such behaviors belong to the class of systems that exhibit what has come to be referred to as selforganized criticality (SOC) [3]. Examples of SOC are the sandpile, forest fire, earthquake and other large scale problems. In the sandpile problem the distribution of avalanche sizes satisfies a scaling law, just like (1), and is universal [3]. The use of cellular automata and the finding of (11) for quarkhadron phase transition put the physics of heavy-ion collisions for the first time in touch with modern trends in statistical physics.

There are two ways in which the rules in [2] can be improved. So far the clusters formed are rigid and have irregular shapes, whereas the possibility to deform seems reasonable. Furthermore, the breakup of a cluster should be allowed, as is done in 1D [1], but too complicated to implement in 2D [2]. Both of these improved features have been introduced recently in a new cellular automaton, in which a cluster is treated as a vertical stack located at one site, having an effective radius of extension

$$
R=\alpha \sqrt{S / \pi}
$$

where $\alpha$ is a number that parametrizes the shape of the cluster [4]. A cluster with $\alpha>1$ would be dendritic, while $\alpha=1$ corresponds to a circle. Breakup $\left(S \rightarrow S_{1}+S_{2}\right)$ is allowed to take place with the probability

$$
B\left(S, S_{1}, S_{2}\right)=\beta\left(\sqrt{S_{1} / S}+\sqrt{S_{2} / S}\right)^{-1}
$$

where $\beta$ is another parameter. We have found that when $p>0.05, P(S)$ is insensitive to the value of $\alpha$ for $1.0<\alpha<1.4$. The dependence on $\beta$ is shown in Fig. 1(d). Evidently, not only is the scaling law unchanged, there is virtually no dependence on the breakup parameter $\beta$, when $p$ is not too small. It means that with sufficient nucleation and growth the broken pieces can readily recombine to form larger clusters.

The clusters that we have considered are produced at the edge of the mixed-phase region still at the transition temperature. What changes can take place as they cool to $T=0$ on their way to the detector is a different issue that cannot be addressed adequately here. If there is no hadron gas surrounding the plasma, there would be no collisions among the clusters, which 
then simply travel in free streaming toward the detector. To separate the various clusters experimentally, we suggest that the $p_{T}$ space be partitioned into many bins so that in each $p_{T}$ bin one analyzes the particle density in small bins in the $\eta-\phi$ space in search for clustering. In that way the various clusters produced in an event do not overlap and defeat identification. Of course, it is necessary to make event-by-event analysis, lest all signatures would be smeared out in an inclusive distribution.

In summary, our theoretical study has led to the result that a universal scaling behavior of cluster production is a likely feature at the end of the quark-hadron phase transition. The important condition under which this result follows is that there is an extended region of mixed phase that persists for a long time. Apart from that, there is approximate independence on all other experimental conditions. The existence of large scale fluctuations in extended systems is just the feature that was discovered in the study of selforganized criticality. Our finding therefore connects heavy-ion collisions to a very new area of research in statistical physics. Although only theoretical at this stage, the result should provide sufficient motivation to search exper-

imentally for the clustering of hadrons, the discovery of which in the data would undoubtedly be a stimulating advance in the creation of quark-gluon plasma.

\section{Acknowledgment}

This work was supported in part by the U.S. Department of Energy under Grant No. DE-FG06-91ER40637, and by the Natural Science and Engineering Council of Canada and by the FCAR fund of the Quebec Government.

\section{References}

[1] R.C. Hwa, C.S. Lam and J. Pan, Phys. Rev. Lett. 72, 820 (1994).

[2] R.C. Hwa and J. Pan, Phys. Rev. C 50, 2516 (1994).

[3] P. Bak, C. Tang, and K. Wiesenfeld, Phys. Rev. A 38, 364 (1988).

[4] R.C. Hwa and J. Pan, OITS-567 (1995). 


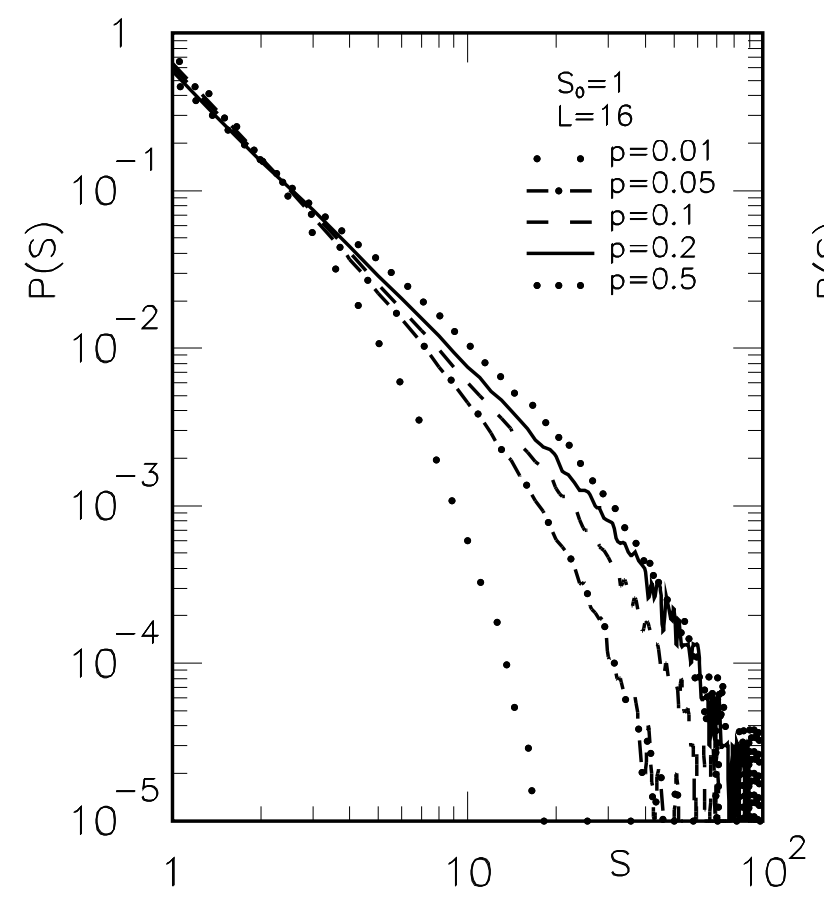

(a)

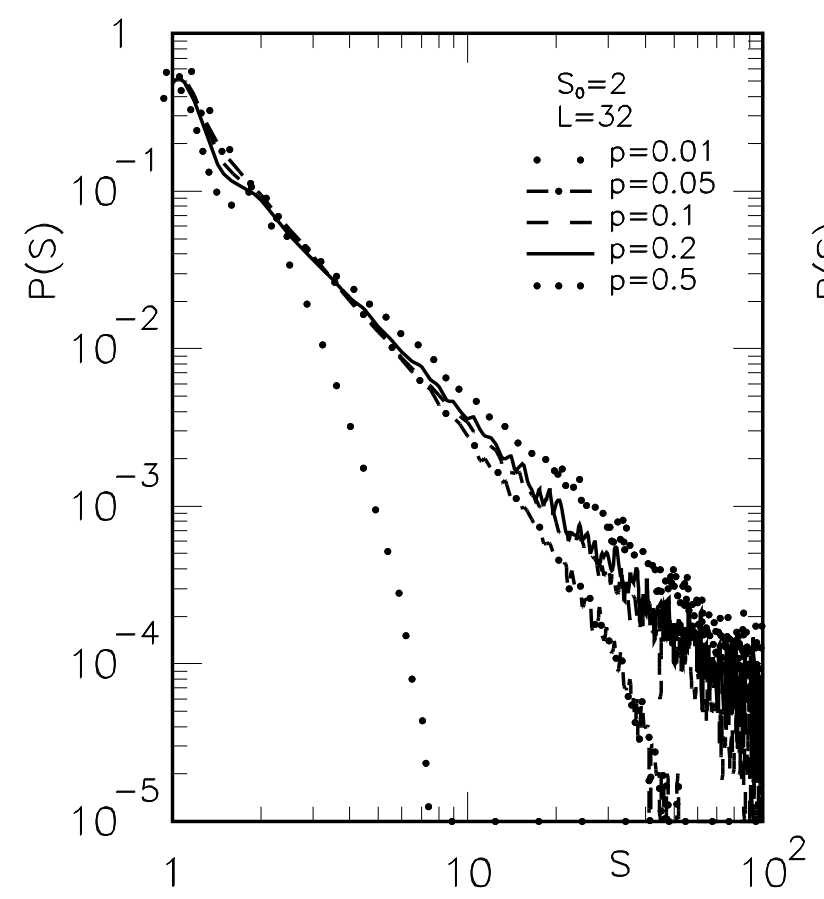

(c)

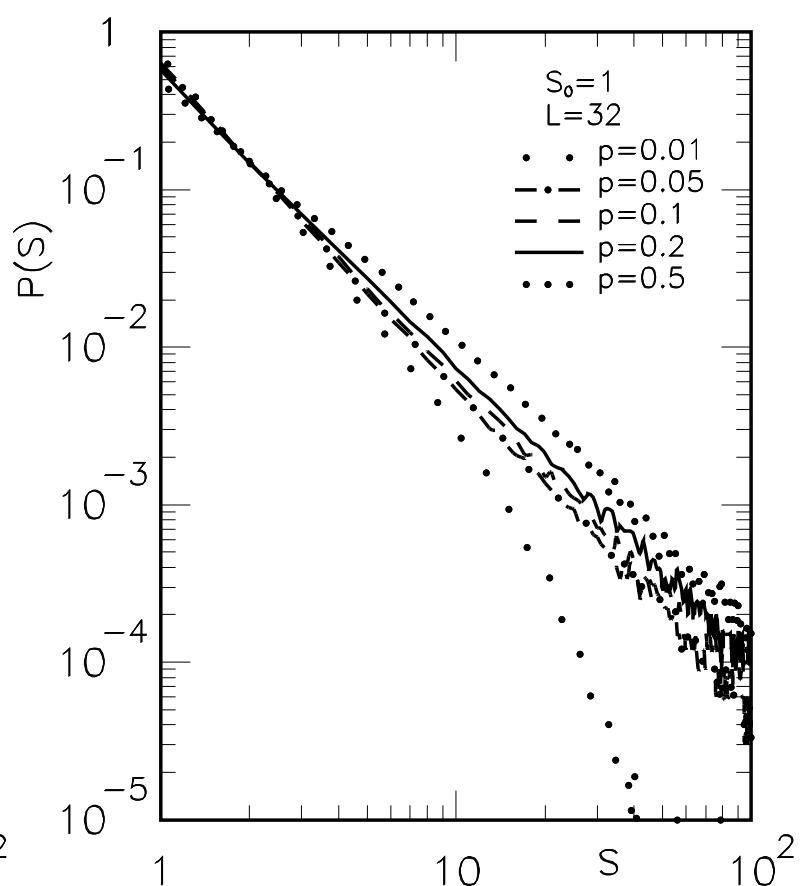

(b)

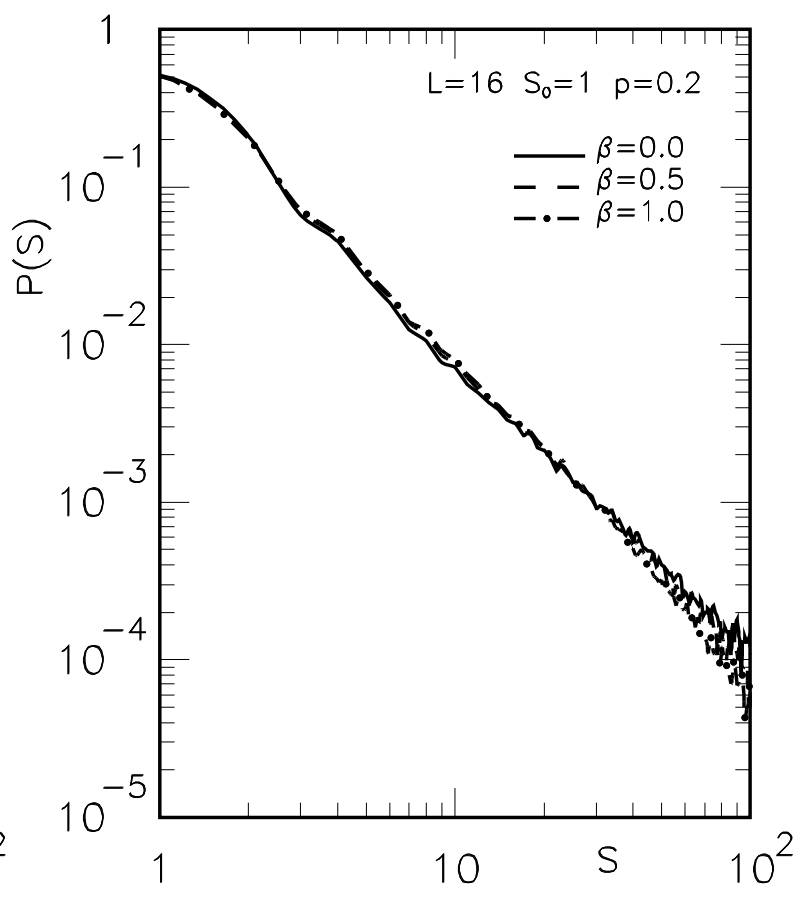

(d)

Fig. 1 